\section{Macular Receptors}

\section{Definition}

Labyrinthine receptors located within the maculae of the utriculus and sacculus whose cilia are embedded in a gelatinous structure (the otolith membrane). The membrane contains crystals of calcium carbonate whose density is three times higher than that of endolymph. Linear accelerations imposed on the head or changes in head position with respect to the vertical axis displace the membrane and stimulate the receptors.

\section{- Peripheral Vestibular Apparatus}

\section{Magnetic and Electric Senses}

\author{
Wolfgang Wiltschko ${ }^{1}$, Bernd Kramer ${ }^{2}$ \\ ${ }^{1}$ Universität Frankfurt, Zoologisches Institut \\ Biologie-Campus der Universität, Frankfurt am Main, \\ Germany \\ ${ }^{2}$ Institut für Zoologie, Animal Behaviour and \\ Behavioural Physiology Research Group, Universität \\ Regensburg, Regensburg, Germany
}

\section{Synonyms}

Magnetoreception; Magnetoperception; Magnetosensitivity; Magnetic orientation; Magnetotaxis; Magnetonavigation; Electroreception; Electric sensitivity; Electric orientation; Electrolocation; Electrocommunication

\section{Definition}

Many animals use magnetic or $>$ electric fields to obtain information about their environment. Although the senses for both types of fields are discussed here in one chapter, the role of these physical features in animal behavior is fundamentally different. The magnetic field used is the -geomagnetic field; it provides a reliable source of information utilized by the animals in numerous ways for orientation in space and possibly time. The electric information used by animals, on the other hand, is normally not provided by the $>$ physical environment, but comes from other animals or is produced by the user of the information for active electrolocation. The - electroreceptor organs of marine elasmobranch fish, termed - ampullae of Lorenzini, might additionally act as magnetoreceptors. At the same time, while the ecophysiology of magnetic and electric senses is generally known, at least in the most prominent animal groups studied, our knowledge about the neurobiological base differs greatly. The neurobiology of the electrical sense is comparatively well understood, and a prominent example of neurobiology, whereas our knowledge on the neurobiology of the magnetic senses is still rather limited; here, most of our knowledge comes from behavioral experiments.

\section{Magnetic Senses}

For animals that are able to perceive magnetic parameters, the geomagnetic field provides an omnipresent source of navigational information. Its field lines exit the earth at the southern magnetic pole, run around the globe and reenter at the northern magnetic pole. This vector quality provides directional information to be used as a magnetic compass, whereas total $>$ intensity and/or $>$ inclination, showing a gradient from the poles to the equator, can provide positional information on a large-scale >magnetic "map" to be used for navigation. The latter two magnetic parameters may also serve as " $>$ sign posts" or triggers, marking specific locations or regions where they elicit specific responses. In a similar sense, even magnetic anomalies could serve to characterize a specific location. Furthermore, the daily variation of the geomagnetic field - in the temperate zones a decrease of magnetic intensity from sunrise to noon, followed by a corresponding increase - have been discussed as potential Zeitgebers for the circadian clock. In summary, in order to make optimal use of the wealth of information offered by the geomagnetic field, animals need sensors for magnetic direction as well as sensors for magnetic intensity or intensity changes.

The use of the magnetic field for locating directions is rather widespread among animals. The associated behavior can be classified either as alignment responses or as a magnetic compass orientation. Alignment responses are characterized by an axial preference of the prominent magnetic directions, with the dances of honeybees on a horizontal comb being a classic example: without view of the sky, the largest activity is found along the magnetic $\mathrm{N}-\mathrm{S}$ and the $\mathrm{E}-\mathrm{W}$ axes (Fig. 1).

When, however, the magnetic field is used for compass orientation the animals can prefer any arbitrary direction with respect to the direction of the magnetic field. The behavioral context of the animal determines the specific angle relative to magnetic north. The selected course, or "set direction," of the compass can be of different origin: it can be innate, imprinted or learned.

The best studied examples for magnetic compass orientation is that of birds (Fig. 2).

It is a so-called " $>$ inclination compass," as the birds do not use the polarity of the magnetic field, but the inclination of the magnetic field lines in space to derive directions. This type of compass response does not distinguish between magnetic North and South, but between "poleward," where the axis of the field lines forms the acute, and "equatorward," where it forms the 
obtuse angle with gravity. At the same time, the avian magnetic compass functions only in a narrow $>$ intensity window; this functional window is finely tuned to the ambient magnetic intensity where the birds live. These findings from behavioral experiments imply specific

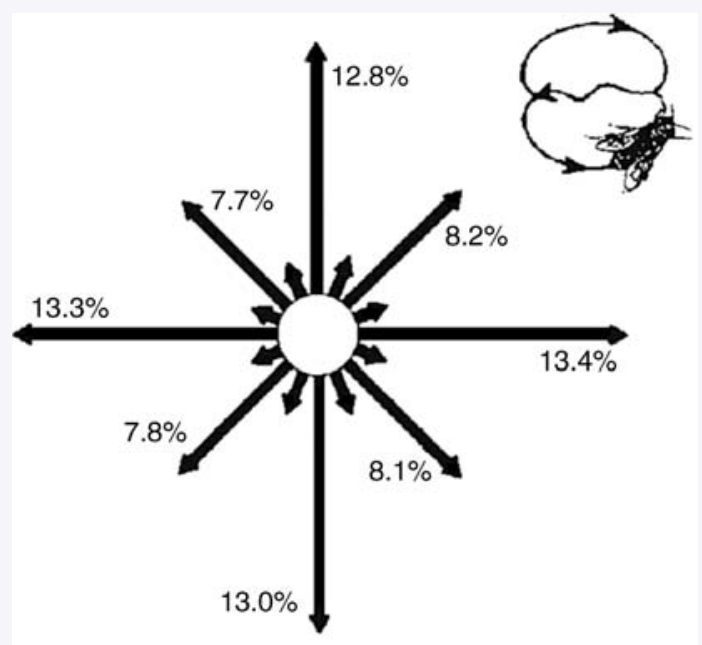

Local geomagnetic field

Magnetic and Electric Senses. Figure 1 Dancing directions of bees on a horizontal comb in the absence of directing visual stimuli under diffuse light in the natural geomagnetic field. 24,601 individual dances were recorded; the arrows are proportional to the percentage of dances in the various directions. For the main classes the percentage is given numerically. The results clearly demonstrate a spontaneous preference of the main-direction $\mathrm{N}, \mathrm{E}, \mathrm{S}, \mathrm{W}$ and a still remarkable preference of the intermediate direction NE. SE, SW, NW (after [37]). characteristics of the receptors mediating magnetic compass orientation in birds. Various other animals are also able to perceive the direction of the magnetic field and use it as a magnetic compass. Two types of compass $\checkmark$ mechanisms have been described: marine turtles and amphibians have an inclination compass like birds, but mole rats, some fish and all invertebrates studied so far use a " $>$ polarity compass" that is based on the polarity of the magnetic field lines, similar to our technical compass. This suggests the existence of at least two different receptor mechanisms among vertebrates [1].

Birds use their magnetic compass for homing and for migration. Avian navigation is usually described as a two-step process: the direction to the goal is first determined as a compass course (= set direction); then, this course is located with a compass and converted into a heading for flight. Orientation within the home range and homing means that the compass course varies according to the position of the animal relative to home; it is determined by a navigation process or remembered from previous visits. For migration, the migratory direction as fixed set direction is innate; the respective course is genetically encoded and passed on from one generation to the next. Here, the geomagnetic field serves as external reference system and, together with celestial rotation, ensures that this genetic information is converted into the species-specific migratory direction [2]. In birds, the magnetic compass is also involved in establishing the directional relationship between sun azimuth and the circadian clock for the sun compass and in calibrating a stellar compass for nocturnal migrants. The biological significance of the avian magnetic compass lies in its role as a basic component of a complex navigational system (see [1]).

In non-avian species, magnetic compass orientation also provides a directional reference in various

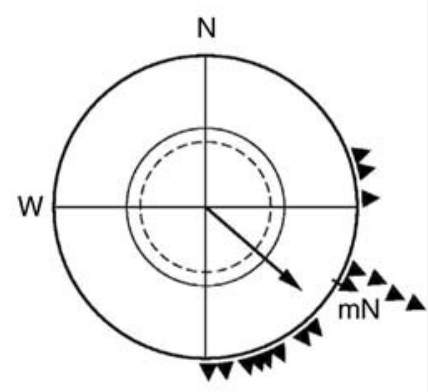

Magnetic and Electric Senses. Figure 2 Orientation behavior of 16 European Robins in spring, tested in the natural geomagnetic field (left) and in an experimental magnetic field with the horizontal component shifted to the $\mathrm{SE}($ right). $\mathrm{mN}$, magnetic North. The triangles at the periphery of the circle mark the headings of the individual birds. The arrows represent the mean vectors calculated from the 16 headings. The length of the vector is proportional to the radius of the circle. The two inner circles are the $5 \%$ (dashed) and the $1 \%$ significance levels of the Rayleigh test (after [35]). 
behavioral situations. It is used by salmon fry that prefer innate directions to find their way in complex lake-river systems [3] and by hatchlings of sea turtles that use an imprinted magnetic course to head away from the shore to the open sea. Another famous example is the wellstudied "y-axis"- orientation that is typical for animals living at the border of land and water, e.g., salamanders, beach hoppers and isopods: here, the animals move along a magnetic axis perpendicular to the shoreline toward water or land, depending on their physiological needs (Fig. 3; [4]).

In birds as well as in other vertebrate species, the magnetic compass may be used in a navigational strategy called "path integration," based on directional information obtained during the outward journey. Recording the net direction of the displacement could be based on endogenous (idiothetic) cues alone, or on external (allothetic) factors. In very young pigeons, wood mice, box turtles and young alligators, the geomagnetic field was shown to provide the external reference for path integration (see [1]). Blind mole rats of the genus Spalax were shown to use idiothetic factors alone over short distances, but to turn to the magnetic field as external reference on more extended excursions [5].

A special case of directional orientation with the help of the magnetic field is found in $>$ magnetic bacteria. While all animals detect the direction of the magnetic field with specialized receptors and act according to this information, magnetic bacteria contain chains of tiny crystals of magnetic material. By the force of the geomagnetic field lines, they are passively rotated and aligned along the field lines by magnetic crystals; they then propel themselves along the field lines down into the mud (see [6]). Their orientation is thus fundamentally different from that of animals.

The geomagnetic field is not only used as a source of directional information. An increasing body of evidence suggests that non-directional features of the geomagnetic field are also utilized by animals. Because of the gradients running from the poles to the equator, magnetic intensity and/or inclination can serve as components in a potentially world-wide $>$ navigational map. The use of magnetic "map"-factors has been discussed for birds since the nineteenth century and is indicated by some findings with homing pigeons, other birds, marine turtles and alligators; Fig. 4 gives a recent example of navigation based on magnetic factors in an invertebrate for the first time.

Navigational "maps" are typical for territorial animals; their biological function is to ensure that the animals find back to their home territory after extended excursions or displacements. Such "maps" are established by experience, involving learning and memory. In these learning processes, the magnetic compass might be involved as reference system, allowing animals to record the regional directions in which navigational factors show a maximum change.

Aside from their role as components of the navigational "map," magnetic intensity and inclination or a combination of both may serve as "sign posts" or triggers to elicit specific preprogrammed responses in certain areas characterized by these magnetic features. A famous example is the responses observed in young sea-turtles from Florida that spend their first year of life

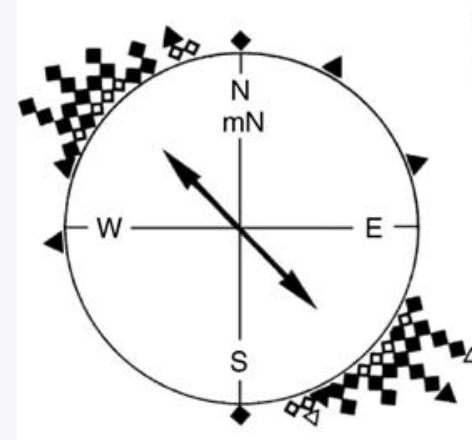

Geomagnetic field

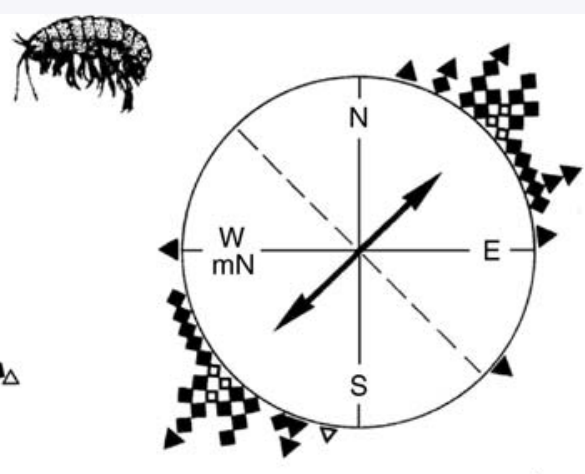

Magnetic north in geographic west

Magnetic and Electric Senses. Figure 3 Orientation of the equatorial sandhopper Talorchestia martensii in the laboratory in a centrally lit arena. Test in the local geomagnetic field (left); test with magnetic North shifted $90^{\circ}$ counterclockwise to geographic west (right). The theoretical escape direction to the sea of $145^{\circ}$ is marked by the arrowhead outside the circle; the symbols at the periphery indicate the mean headings of individual sandhoppers: triangles unimodal behavior; diamonds axially bimodal behavior, with both ends of the axis indicated; solid symbols samples significant according to Rayleigh test; open symbols non-significant samples. The double-headed arrows represent the mean axes, the dashed diameter in the right diagram marks the axis of the respective controls (after [4]). 


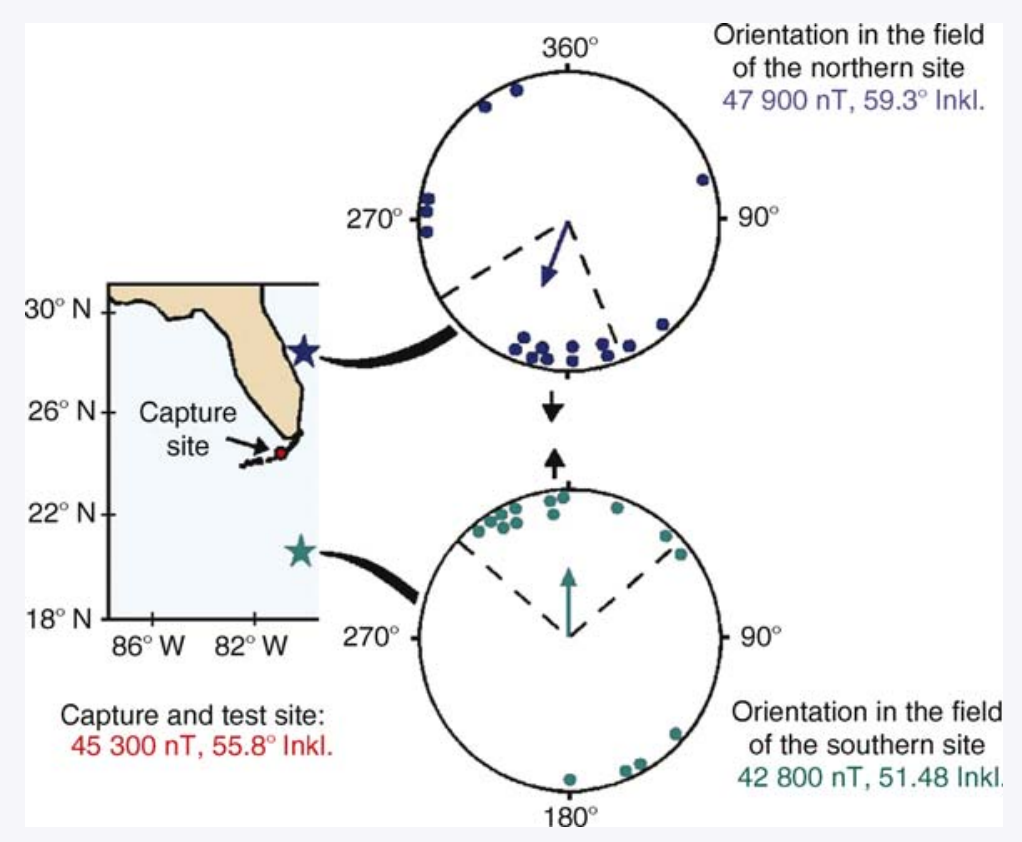

Magnetic and Electric Senses. Figure 4 True navigation by magnetic parameters indicated in spiny lobsters. The lobsters were tested near their capture site in magnetic fields replicating the ones of two distant geographic locations (marked with asterisks). In the circular diagrams, the small arrows outside of the circle indicate the home directions from the simulated sites. Dots at the periphery of the circle mark the headings of single lobsters; the arrow represents the mean vector proportional to the radius of the circle, with the dashed radii indicating the $95 \%$ confidence interval of the mean direction (after [19]).

in the Atlantic gyre. When freshly hatched turtles were exposed in the laboratory to magnetic fields simulating those at the edge of the gyre, they altered their headings and chose directions that would have brought them toward the center of the gyre, thus helping them stay within suitable waters (see " "Entry" Magnetic maps). Similar preprogrammed responses have also been described for migratory birds that change direction or respond physiologically by increasing their fat load when encountering specific magnetic conditions.

A further role of the magnetic field in orienting animal movements has been discussed for the extended migrations of whales between their polar feeding grounds and the temperate regions where they spend the winter; following magnetic contours was suggested as navigational strategy (see [1]).

Organisms like magnetic bacteria need no perception mechanism at all, as they are passively aligned by the force of the geomagnetic field. In contrast, animals that actively respond to magnetic parameters need to obtain information about the direction or the intensity of the geomagnetic field. By what kind of mechanism they detect these features is not yet entirely clear, however. Experiments with birds suggest that magnetic compass orientation requires a stable direction of the magnetic field, but would tolerate changes in intensity and inclination to a certain degree. For the magnetic components of the navigational "map," on the other hand, detecting minute changes in intensity is crucial, whereas the direction of the field appears not to be important. Considering the physical aspects of magnetoreception, three mechanisms have been suggested: induction, $>$ magnetite-based mechanisms and $\checkmark$ radical-pair processes.

Electric current induction through the Faraday Effect as primary process for magnetoreception is an option only for marine fish. It has been discussed for elasmobranch fish, because the ampullae of Lorenzini, the electroreceptor $>$ organs of sharks and rays (see below), are highly sensitive to electric fields. With a threshold of as low as $5 \mathrm{nV} / \mathrm{cm}$, these receptor organs are, at least theoretically, sensitive enough to distinguish the voltage induced by the normal swimming speed when moving in different directions and thus provide the information required for magnetic compass orientation (see [1]).

-Magnetite is a specific form of $\mathrm{Fe}_{3} \mathrm{O}_{4}$, with its magnetic features depending on particle size. Small crystals of less than $40 \mathrm{~nm}$ are superparamagnetic (SPM), which means that they do not have a stable magnetic moment; their magnetic moment fluctuates due to thermal instability, but can be stabilized by static ambient magnetic fields like the geomagnetic field. Particles of an intermediate size of 40-120 nm form 
so-called single domains (SD) and have a stable magnetic momentum; theoretically, they could align themselves along the magnetic field lines like minute compass needles. Larger particles above $120 \mathrm{~nm}$ become multi-domain and have no pronounced net magnetic moment, because the moments of the various domains largely cancel each other.

Considerations on magnetite-based magnetoreception usually favored single domains, and several models have been suggested how single domains could mediate magnetic information. E.g., attached to hair cells or specialized membranes, they would be able to transduce the magnetic torque to mechanical torque and thus act as a compass. By magnetic remanence measurements, single domain particles were indicated in the tissues of numerous animals (see [7]). In vertebrates, magnetite was found in the nasal and orbital region, a region which is innervated by the ophthalmic branch of the nervus trigeminus, from which electrophysiological responses to magnetic stimulation involving changes in intensity were recorded. Structures that may be candidates for a $>$ magnetite-based magnetoreceptor based on single domains have been described for fish (e.g. [8]). In the upper beak of homing pigeons a structure has been identified which contains clusters of superparamagnetic magnetite; it may serve as modified pressure receptor measuring magnetic intensity [9].

The radical-pair hypothesis postulates magnetoreception by specialized photopigments. By photon absorption, molecules are raised to the excited singlet state, where some of them undergo a transition into the excited triplet state. The probability to reach the triplet state, and with it, the triplet yield, depends on the alignment of the photopigments with respect to the magnetic field lines. In the spherical or hemispherical structure, the triplet yield would form a specific pattern that was centrally symmetric to the axis of the field lines, thus forming a $>$ chemical compass. By comparing the triplet yield in the various spatial directions, animals could obtain information on the direction of the magnetic field [10]. Because of their biochemical properties, cryptochromes are discussed as possible candidates for the photopigments underlying these processes. The radical-pair model is indirectly supported by behavioral findings indicating that the magnetic compass of salamanders and birds is light-dependent: short-wavelength monochromatic light allows normal orientation, whereas the use of long-wavelength light abolishes orientation behavior [11]. The recent observation that the magnetic compass of birds can be disrupted by high-frequency magnetic fields points directly to a radical-pair process underlying magnetoreception [12].

In salamanders, the receptors mediating compass information appear to be situated in the pineal, the ancestral "third eye" of vertebrates [13]. In birds, too, magnetosensitive cells have been found in the pineal; however, in two species of passerine birds, magnetoreception was shown to occur almost exclusively in the right eye [14]. Since the optic nerves of birds cross over almost completely, this means that magnetic information is processed predominantly in the left hemisphere of the brain. The tectofugal part of the visual system shows a marked anatomical lateralization, with the relevant pathways in the left hemisphere more pronounced than in the right [15] which might also be associated with processing magnetic compass information. Electrophysiological recordings from the tectum opticum and the nucleus of the basal optic root (nBor), a nucleus belonging to the tectofugal system, revealed units that were stimulated by changing the direction of the ambient magnetic field [16].

Altogether, the available findings on magnetoreception suggest a variety of mechanisms based on different principles. It seems plausible to assume that the magnetic compass and the magnetic part of the "map" require different types of magnetoreceptors, because they utilize different physical features of the magnetic field. This asks for different primary processes and different ways of neuronal processing. So far, evidence from birds suggests that a $>$ radical-pair mechanism in the right eye provides compass information, whereas magnetite-based receptors associated with the trigeminal system provides intensity information for the "map." A radical-pair mechanism is also discussed for the compass of salamanders, but what kind of mechanisms other animals might use is not yet known.

\section{Electric Senses}

In contrast to the geomagnetic field, the electric field of the earth is highly variable. Its variability is caused by differences in the activity of thunderstorms and related phenomena, and the $>$ field intensity depends on many factors like air humidity, temperature and conductivity of the surface. The polarity of the electric field is directed vertically downward. In view of this, it is hard to see how the electric field could provide information that is useful to animals. Yet a number of animals were found to be electroreceptive. Only in the past 50 years, peculiar structures (sensory pores) in the skin of some aquatic vertebrates that had been known for a long time were identified as electroreceptor organs (see entry "Electroreceptor organ"). Such electroreceptor organs are found in all groups of lower aquatic vertebrates and in certain amphibians, while they are lacking in most of the modern fish (such as the Teleostei within the Neopterygii). In two, possibly three, not closely related lineages of Teleostei, they obviously reevolved independently, namely in the $>$ Mormyriformes from the Osteoglossomorph branch and the $>$ Siluriformes (catfish) and $>$ Gymnotiformes from the Neognath branch. Because of the insulating properties of air, 
electroreceptor organs are generally lacking in terrestrial vertebrates such as reptiles, birds and mammals, the only exceptions being the Monotremata, the $\checkmark$ Echidna and the $>$ Platypus, where electroreceptor organs have been recently described (see entry " $\triangleright$ Electroreception and Electrolocation in the platypus and the echidna").

Electroreceptor organs are voltmeters; the receptor cells are modified hair cells and are part of the $>$ octavolateral sensory system that is responsible for hearing and the sense of equilibrium. Electroreceptor organs are contacted by sensory nerves only.

There are several types of electroreceptive organs. -Ampullary electroreceptor organs are extremely sensitive to weak gradients of the electric field. The ampullae of Lorenzini of the marine elasmobranch fish have thresholds of about $5 \mathrm{nV} / \mathrm{cm}$, the analogous " $>$ ampullary" receptor organs of freshwater teleosts may reach $1-5 \mu \mathrm{V} / \mathrm{cm}$ maximum sensitivity. Reflecting their different origin, the structure of ampullary electroreceptor cells differs in the various groups: in non-teleosts, they bear a kinocilium, sometimes in addition to microvilli, whereas in electroreceptive teleosts, the secondarily evolved electroreceptor cells have only microvilli, but no kinocilium (Fig. 5).

The ampullary organs as a whole, as well as their teleost analogs, consist of a layer of receptor cells lining an ampulla which is embedded in the skin and connects to the outside by a canal. In marine fish, this canal is long, in fresh-water fish, it is short. The adequate stimulus is the voltage difference between the inside of the skin and the surface of the fish. The lumen of the ampulla and of the canal connecting to the surface is filled with a highly conductive jelly so that the electric potential at the lumenal surface of the receptor cells is almost identical with that on the outside of the skin. Because of the relatively high conductivity of the skin of marine fish, their ampullary canals must be longer than those of freshwater fish to achieve similar sensitivity. Also, whereas the ampullary organs of the ancestral forms of vertebrates are stimulated by negative voltages on the outer skin, those of teleosts are stimulated by positive voltages.

Animals with ampullary organs make use of their high sensitivity to detect minute changes in the ambient electric field, thereby locating prey by detecting the normal electric activity of living organisms, like prey buried in sand or active at night. The detection of electric fields that are produced by other organisms rather than the animal itself is called passive electrolocation. Orientation responses along local electric fields, even magnetoreception based on the voltage induced by moving in various directions with respect to the geomagnetic field have been described (see above).

Most $>$ Electrogenic fishes are not only sensitive to ambient electric fields, but also generate their own

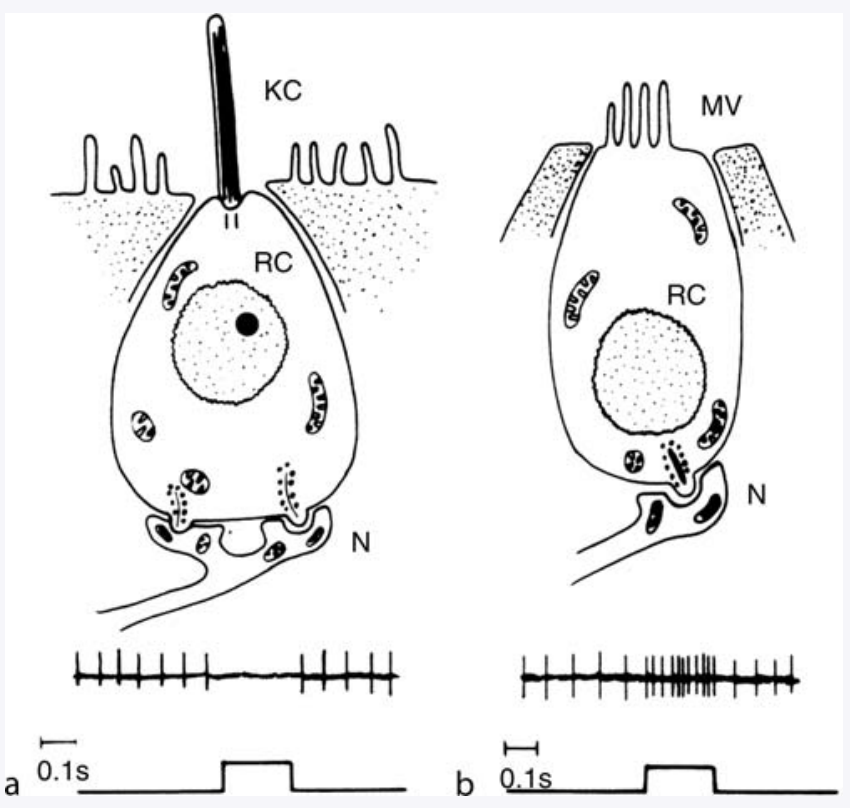

Magnetic and Electric Senses. Figure 5 Ampullary electroreceptor cells $(R C)$ of nonteleosts (a) bear an apical kinocilium $(K C)$, sometimes in addition to microvilli $(M V)$, whereas electroreceptive teleosts (b) have only microvilli and no kinocilium. The spontaneously active, afferent nerve fibers $(N)$ increase their firing rate when the electrical stimulus (in this case a square-wave pulse of $200 \mathrm{~ms}$ ) is positive outside the ampulla in teleosts, while in nonteleosts a negative stimulus is required for a similar response (from [36] modified). 
electric fields by specialized $\triangleright$ electric organs (see entry "Electric fish"). Any living tissue generates \electric fields of low intensity by maintaining the ionic balance of its cells, and by field potentials from nerve and muscle activity; these fields' strength ranges from $0.5 \mathrm{mV}$ to several $\mathrm{mV}$ (as measured between the body and a distant electrode). Electrogenic fish, however, possess electric organs consisting of orderly arranged, closely packed groups of modified muscle cells (nerve endings in one taxon). These fishes can discharge their electric organs in a controlled way; thereby producing electric fields ranging from a few $\mathrm{mV}$ up to $500 \mathrm{~V}$. Electrogenic fish are found among both cartilaginous and bony fishes; prominent examples for species producing strong electric fields are the electric rays (genus Torpedo) and the electric eel (Electrophorus electricus).

The rhythmic discharge of electric organs ( $\triangleright E O D)$ generates species-characteristic electric signals that are highly stereotyped (see entry " $>$ Electric organ discharge"). Among the teleosts, both gymnotiform and mormyriform fish have a second type of electrosensitive receptor organ besides the ampullary organs, namely tuberous electroreceptor organs. These receptor organs are specifically tuned to the frequency spectrum (15-20,000 Hz, depending on the species) generated by the discharge of their own electric organ.

The tuberous electroreceptor organs occur in two types: one acts as $>$ time marker unit of high sensitivity and short and constant latency, whereas the other type acts as amplitude coder that is relatively insensitive in absolute terms, but encodes minute changes in the intensity of the fishes' own electric organ discharges. Like ampullary receptor organs, tuberous receptor organs are located in the skin and form part of the -lateral line system whose afferences project to the -electrosensory lobe of the lateral line (ELL). With these tuberous electroreceptor organs, the fish detects impedance inhomogeneities in its environment by recording the associated modulations of their electric organ discharges in amplitude and phase. Thus, these fishes are capable of active electrolocation. Probably the predominant function of producing and perceiving electric fields is >electrocommunication. With their electric organs, fishes produce signals either of the pulse- or the wave type that can be perceived and decoded by their neighbors (see entry "Electrocommunication and Electrolocation").

The tuberous electroreceptor organs of the African Mormyridae are the $>$ knollenorgane and the $>$ mormyromasts (Fig. 6).

Knollenorgane fire one action-potential per EOD pulse. As the mormyrid fish brain blanks the reafferences from self-generated electric organ discharges, only the pulses from other fish gain access to the higher centers of the brain. Knollenorgane thus serve electrocommunication. The second type of tuberous electroreceptor organ, the mormyromasts, has a higher threshold and therefore responds primarily to the fish's own EODs (those of other fish being centrally blanked); they appear to be primarily responsible for active electrolocation. Mormyromasts comprise two kinds of electroreceptor cell that are innervated separately. Mormyromasts are capable of coding for both resistive
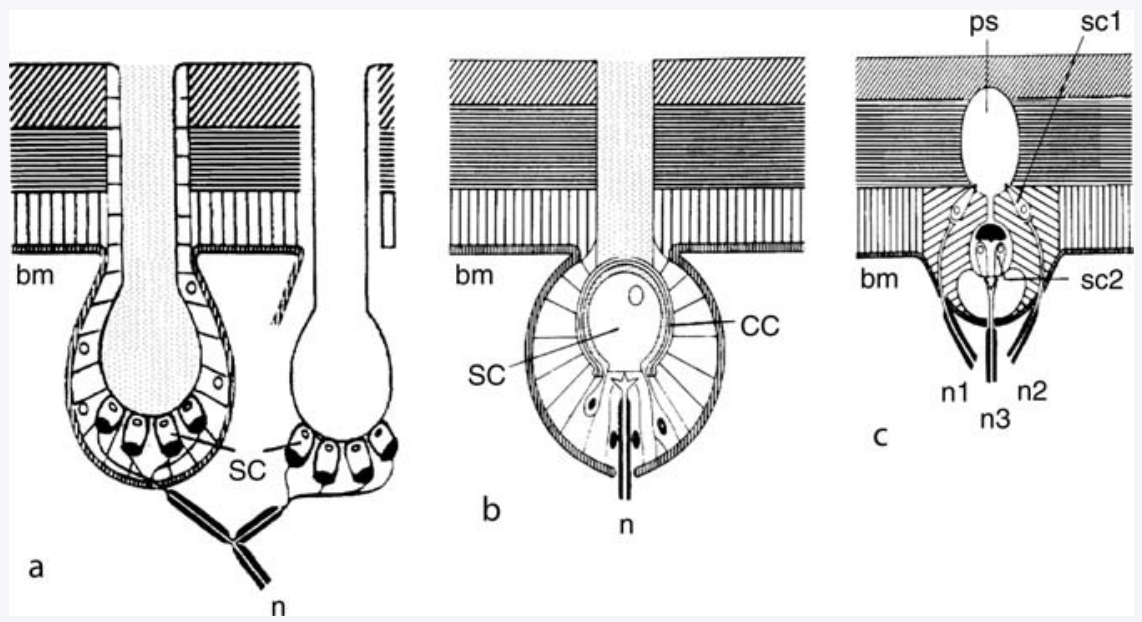

Magnetic and Electric Senses. Figure 6 Schematic electroreceptor organs in freshwater teleosts, located in invaginations (or ampullae) of the epidermis. (a) Small pit organ, the teleost equivalent of the ampullary electroreceptor organ of similar structure that is common to all classes of lower aquatic vertebrates (but lacking in neopterygians, including teleosts). (b) Knollenorgan, one kind of tuberous electroreceptor organ present in mormyrid fish. (c) Mormyromast, the other kind of tuberous electroreceptor organ in mormyrid fish [33]. 
and capacitive loads associated with nearby objects, and fish can discriminate between live and dead material by their difference in capacitive impedance.

The central-nervous processing of information from the various types of electric organ takes place in different structures of the brain; e.g., the input from mormyromasts and knollenorgane, involving active electrolocation and $>$ communication, are processed separately. In gymnotiform fishes, many of which generate discharges of the wave type, electrosensory reafferences are encoding time (phase) and amplitude (of the wave discharge envelope) by different receptor organs whose afferences are processed separately by different parts of the brain, analogous to the hearing system of other vertebrates. This separate neuronal processing of phase- and amplitude information is thought by one theory (theory I) to be important for the so-called " $>$ jamming avoidance response" (JAR) that was mainly studied in the gymnotiform wave fish Eigenmannia virescens. A fish will tend to shift its own discharge frequency away from a stimulus too close in frequency such that the two signals beat against each other faster, in an attempt to restore its active electrolocation performance (according to theory I; see entry $\triangleright$ Temporal coding in electroreception). By contrast, theory II stresses that the jamming avoidance behavior has long been shown to be sexually dimorphic and to differ between juveniles and adults, and envisions its sensory mechanism and functional adaptation to be radically different (for aspects of theory II, see also entry $>$ Electric communication and electrolocation). For example, incompatible with theory I is the observation that the JAR threshold is identical to stimulus detection threshold, that is, it is defined by the more sensitive $>\mathrm{T}$ receptor organs. Consequently, phase (timing) information (that is coded for by the $>\mathrm{T}$ receptor organs) has been found to be both necessary and sufficient for evoking and guiding the JAR, and information on amplitude modulation of the beat envelope from the rather insensitive $P$ receptor organs is not available in the threshold range and well above (as would be required by theory I, but not theory II; P receptor organs or probability coders report the amplitude modulation of the EOD by a stimulus of similar frequency only at sufficiently strong modulation depth, that is, sufficiently strong stimulus/EOD intensity ratios). Furthermore, the JAR may be evoked by a stimulus of exactly the fish's own frequency (that is, the frequency difference equals $0 \mathrm{~Hz}$ ), even when maintained dynamically constant by a frequency clamp; an observation theory I cannot explain but which does not present a problem for theory II. According to theory II, by purely temporal analysis of beat features, the zerocrossings times of the individual oscillations within a beat wave that are reported by $\mathrm{T}$ receptor organs, the fish extracts (i) the strength of the stimulus signal harmonic closest to its own fundamental discharge frequency (only that stimulus harmonic is driving the JAR), (ii) the frequency difference and its sign, (iii) the waveform of the stimulus signal. The JAR is thought by theory I to support active electrolocation in the presence of "jamming" conspecifics in the near field (the reach of active electrolocation is limited to a few $\mathrm{cm}$ ). Theory II, however, has demonstrated that the JAR supports electrocommunication and the detection of EOD waveforms generated by neighbors from a much greater distance (far field sensitivity). This discussion exemplifies the dual nature of the electric system that is adapted for both functions.

\section{References}

1. Wiltschko R, Wiltschko W (1995) Magnetic orientation in animals. Springer, Berlin Heidelberg New York

2. Weindler P, Wiltschko R, Wiltschko W (1996) Magnetic information affects the stellar orientation of young bird migrants. Nature 383:158-160

3. Quinn TP (1982) Intra-specific difference in Sockeye Salmon fry compass orientation mechanisms. In: Brannon EL, Salo EO (eds) Proceedings of the Salmon and Trout Migratory Behavior Symposium, University of Washington, Seattle, pp 79-85

4. Pardi L, Ugolini A, Faqi AS, Scapini F, Ercolini A (1988) Zonal recovering in equatorial sandhoppers: interaction between magnetic and solar orientation. In: Chelazzi G, Vannini M (eds) Behavioral adaption to intertidal life. Proceedings of the NATO Science, Plenum, New York London, pp 79-92

5. Kimchi T, Etienne AS, Terkel T (2004) A subterranean mammal uses the magnetic compass for path integration. Proc Nat Acad Sci USA 101:1105-1109

6. Mann S, Sparks NHC, Board RG (1990) Magnetotactic bacteria: microbiology, biomineralization, palaeomagnetism and biotechnology. Adv Microb Physiol 31:125-181

7. Kirschvink JL, Jones DS, MacFadden BJ (1985) Magnetite biomineralization and magnetoreception in organisms. Plenum, New York

8. Walker MM, Diebel CE, Haugh CV, Pankhurst PM, Montgomery JC, Green CR (1997) Structure and function of the vertebrate magnetic sense. Nature 390:371-376

9. Fleissner G, Holtkamp-Rötzler E, Hanzlik M, Winklhofer M, Fleissner G, Petersen N, Wiltschko W (2003) Ultrastructural analysis of a putative magnetoreceptor in the beak of homing pigeons. J Comp Neurol 458:350-360

10. Ritz T, Adem S, Schulten K (2000) A model for visionbased magnetoreception in birds. Biophys J 78:707-718

11. Wiltschko W, Wiltschko R (2002) Magnetic compass orientation in birds and its physiological basis. Naturwissenschaften 89:445-452

12. Ritz T, Thalau P, Phillips J, Wiltschko R, Wiltschko W (2004) Avian magnetic compass: resonance effects indicate a radical pair mechanism. Nature 429:177-180

13. Deutschlander, ME, Borland SC, Phillips JB (1999) Extraocular magnetic compass in newts. Nature 400:324-325 
14. Wiltschko W, Munro U, Wiltschko R, Kirschvink J (2002) Magnetite-based magnetoreception in birds: the effect of a biasing field and a pulse on migratory behavior. J Exp Biol 205:3031-3037

15. Güntürkin O (1997) Morphological asymmetries of the tectum opticum in the pigeon. Exp Brain Res 116:561-566

16. Semm P, Nohr D, Demaine C, Wiltschko W (1984) Neural basis of the magnetic compass: interaction of visual, magnetic and vestibular inputs in the pigeon's brain. J Comp Physiol 155:283-288

17. Markowski B, Baier B, Kramer B (2007) Differentiation in electrical pulse waveforms in a pair of sibling dwarf stonebashers, Pollimyrus castelnaui and P. marianne: possible mechanisms and functions (Mormyridae, Teleostei). Behaviour 145:115-135

18. Bennett MVL (1971) Electroreception. In: Hoar WS, Randall DJ (eds) Fish physiology, vol V. Academic, London New York, pp 493-574

19. Boles LC, Lohmann KJ (2003) True navigation and magnetic map in spiny lobsters. Nature 421:60-63

20. Bullock TH, Hopkins CD, Popper AN, Fay RR (eds) (2005) Electroreception. Springer, Berlin Heidelberg New York

21. Heiligenberg W (1991) Neural nets in electric fish. MIT, Cambridge, Mass.

22. Hopkins CD (1999) Signal evolution in electric communication. In: Hauser MD, Konishi M (eds) Design of animal communication. MIT, Cambridge, Mass., pp 461-491

23. Kramer B (1990) Electrocommunication in teleost fishes: behavior and experiments. Springer, Berlin Heidelberg New York

24. Kramer B (1996) Electroreception and communication in fishes. Gustav Fischer Verlag, Stuttgart, Jena, Lübeck, Ulm

25. Kramer B (1999) Waveform discrimination, phase sensitivity and jamming avoidance in a wave-type electric fish. J Exp Biol 202:1387-1398

26. Ladich F, Collin SP, Moller P, Kapoor BG (eds) (2006) Communication in fishes. Science, Enfield, New Hampshire, USA

27. Lissmann HW (1958) On the function and evolution of electric organs in fish. J Exp Biol 35:156-191

28. Meek J, Grant K, Bell C (1999) Structural organization of the mormyrid electrosensory lateral line lobe. J Exp Biol 202:1291-1300

29. Moller P (1995) Electric fishes: History and behavior. Chapman \& Hall, London

30. Peters RC (1993) Orientation in catfish and dogfish. Orientation and navigation. The Royal Institute of Navigation, Oxford, pp 33-43

31. Scheich H (1977) Neural basis of communication in the high frequency electric fish, Eigenmannia virescens (jamming avoidance response). I. Open loop experiments and the time domain concept of signal analysis. II. Jammed electroreceptor neurons in the lateral line nerve. III. Central integration in the sensory pathway and control of the pacemaker. J Comp Physiol A 113:181-255

32. Stoddard PK, Zakon HH, Markham M, McAnelly L (2006) Regulation and modulation of electric waveforms in gymnotiform electric fish. J Comp Physiol A 192:613-624
33. Szabo T (1974) Anatomy of the specialized lateral line organs of electroreception. In: Fessard A (ed) Handbook of sensory physiology, vol III/3. Springer, Berlin Heidelberg New York, pp 13-58

34. von der Emde G, Schwarz S (2001) How the electric fish brain controls the production and analysis of electric signals during active electrolocation. Zoology 103:112-124

35. Wiltschko W, Wiltschko R (1999) The effect of yellow and blue light on magnetic orientation in European robins, Erithacus rubecula. J Comp Physiol A 184:295-299

36. Bullock TH, Heiligenberg W (eds) (1986) Electroreception. John wiley, New york

37. Martin H, Lindauer M (1977) der EinfluB des Erdmagnetfeldes auf die schwereorientierung der Honigbiene (Apis mellifica). J Comp Physiol 122:145-187

\section{Magnetic Bacteria}

\section{Michael Winklhofer}

Department für Geo- und Umweltwissenschaften Sektion Geophysik, Ludwig-Maximilians-Universität Munich, Munich, Germany

\section{Synonyms}

Magnetotactic bacteria

\section{Definition}

Magnetic bacteria are a morphologically and phylogenetically diverse group of motile Gram-negative prokaryotes. Their common feature is intra-cellular $\checkmark$ ferrimagnetic crystals, made of $>$ magnetite $\left(\mathrm{Fe}_{3} \mathrm{O}_{4}\right)$ or greigite $\left(\mathrm{Fe}_{3} \mathrm{~S}_{4}\right)$, called magnetosomes. Rather than being ingested from food, magnetosomes are synthesized within the cell and are most often arranged in a single chain or two chains, apparently fixed within the cell (Fig. 1).

A chain of magnetosomes carries a permanent - magnetic moment and thus acts as an internal compass needle, rotating the cell into alignment with the geomagnetic field axis. Due to this magnetic torque, magnetic bacteria are constrained to move along magnetic field lines when swimming. They are actively motile rather than being pulled or pushed by the magnetic field. Magnetic bacteria can move both parallel and antiparallel to the magnetic field, corresponding to downward (north seeking) and upward (south seeking) motions in the $>$ geomagnetic field, which has inclined lines of forces except at the magnetic equator, where field lines are horizontal. Their magnetically enforced onedimensional locomotion, makes magnetic bacteria highly 